\title{
A Study to Ascertain the Expression of Aquaporin 4 and Neuropeptide $Y$ in the Jejunal Mucosa Secondary to Traumatic Brain Injury in Humans
}

\author{
Parag Parshuram Patil ${ }^{1}$ Arulselvi Subramanian \\ Deepak Agrawal ${ }^{3}$ \\ 1Department of Laboratory Medicine, Jai Prakash Narayan Apex \\ Trauma Centre, All India Institute of Medical Sciences, \\ New Delhi, India \\ 2Department of Forensic Medicine, Jai Prakash Narayan Apex \\ Trauma Centre, All India Institute of Medical Sciences, \\ New Delhi, India \\ ${ }^{3}$ Department of Neurosurgery, Jai Prakash Narayan Apex Trauma \\ Centre, All India Institute of Medical Sciences, New Delhi, India \\ Indian J Neurotrauma:2020;17:130-138
}

Garima Aggarwal ${ }^{1}$ Sanjeev Lalwani²

\begin{abstract}
Address for correspondence Arulselvi Subramanian, MD, Department of Laboratory Medicine, Jai Prakash Narayan Apex Trauma Centre (JANATC), All India Institute of Medical Sciences, New Delhi 110029, India (e-mail: arulselvi.jpnatc@gmail.com).
\end{abstract}

\begin{abstract}
Introduction Gastrointestinal (GI) dysfunction is a common complication in patients with traumatic brain injury (TBI). Studies in rats have shown alterations in intestinal mucosa to correlate with severity and duration of TBI. There is lack of such evidence in humans. So we intended to find correlation between histopathological changes and expression of aquaporin 4 (AQ4) and neuropeptide $\mathrm{Y}$ (NPY) in jejunal mucosa in post TBI patients.

Materials and Methods Autopsy specimens of jejunum were obtained from patients who had died due to TBI $(n=20)$, patients dying due to traumatic injury other than TBI, and patients who were brought dead (diseased controls $n=20$ ). Abdominal trauma was the exclusion criterion for both. Jejunal specimens were grossly examined and then analyzed histopathologically and graded immunohistochemically for AQ4 and NPY. Unpaired $t$-test was used to compare results.

Keywords

- traumatic brain injury

- aquaporin 4

- neuropeptide $Y$

Results After exclusion, 19 cases and 17 controls were studied. No significant difference was observed in the microscopic findings between cases and controls $(p$-value $=0.70)$. The expression of AQ4 was more in cases $(p$-value $=0.04)$ NPY expression was not significantly different ( $p$-value $=0.93$ ).

Conclusion AQ4 can hence be used as a marker of GI injury post TBI. Histopathological examination cannot distinguish between these changes.
\end{abstract}

\section{Introduction}

Traumatic brain injury (TBI) is a common occurrence in road and railway accidents. Although the exact incidence is unknown, it also is an important occurrence in patients

Presentation at a meeting: (part of the study) $3^{\text {rd }}$ AIIMS Neurotrauma Conference (ANTC)- 28-31 October, 2015 presenting with domestic injuries like fall from height, domestic violence, and so forth. ${ }^{1}$ The severity of this injury affects the overall survival, prognosis, multiorgan failure, morbidity, and mortality in such cases. The exact mechanisms that are involved in this are poorly understood, but inflammation, gastrointestinal (GI) dysfunction, ${ }^{2}$ and increased intestinal permeability ${ }^{3-5}$ occurring secondary to the TBI have been proposed to play a crucial role in the pathogenesis.
DOI https://doi.org/

10.1055/s-0040-1713311

ISSN 0973-0508.
(C)2020 Neurotrauma Society of India
License terms

()(1) $\Theta \circledast$ 
Increased intracranial tension is commonly noted in TBI patients, which could potentially modulate these changes via the brain-gut axis. ${ }^{6}$ GI dysfunction occurs at multiple levels like at the gastric level, duodenal level, jejunal level, and large intestinal level. ${ }^{6}$

Animal models have shown alterations occurring are mainly in the mucosal structure causing reduced absorption of nutrients, increased intestinal permeability, translocation of intestinal bacteria, and endotoxin. ${ }^{7}$ The increased permeability leading to faulty transport of solutes and nutrients across the gut is mainly attributable to the alterations in brain-gut axis mediated through various molecules like aquaporin 4 (AQ4) and neuropeptide Y (NPY) ${ }^{8,9}$ Expression of aquaporins, which are water channels expressed in various tissues mediating entry and exit of water molecules across membranes, has been correlated with various GI disorders like chronic gastritis, gastric cancer, and so forth. ${ }^{10}$ Similarly NPY, which inhibits GI motility and electrolyte secretion in the digestive tract thus modifying the input to the brain, ${ }^{8}$ have also been found raised in TBI induced in animal models.

However, there is lack of clinical studies in humans for the same, as most of these studies have been performed in animal models under controlled conditions. So there is limited evidence of TBI-induced changes in human intestines and the pathways involved in the same.

We intended to understand more about the pathogenesis of TBI induced secondary GI changes. For this we targeted the jejunum, as it is one of the most important surfaces in the GI tract responsible for the absorption of nutrients, coming only secondarily to the duodenum. Another important reason for targeting the jejunum was that there has been very little research on the jejunal changes in such cases, as the maximum amount of research has been focused on the gastric and duodenal changes secondary to TBI. We also intended to find out the expression of AQ4 and NPY in the jejunum by immunohistochemistry, as markers of TBI-induced GI changes.

This study will help in future to develop the means and medications to limit the GI damage in such critically ill patients, thus helping them to improve their prognosis and recuperate to health.

\section{Materials and Methods}

- Study design: It was prospective cohort study in which traumatic head injury patients, older than 16 years, requiring admission to Jai Prakash Narayan Apex Trauma Centre (JPNATC), All India Institute of Medical Sciences (AIIMS), New Delhi, were considered.

- Inclusion and exclusion criteria: Cases included patients dying due to traumatic head injury and death occurring in not less than 24 hours. Controls included patient who had died due to trauma other than traumatic head injury and also patients who were brought dead to the hospital after trauma. In patients with blunt/penetrating injury to the abdomen, autopsy was done 12 hours after the death and any autolysed samples were excluded from cases and control groups of the study.
- Sample preparation: Autopsy sections of small intestine $15 \mathrm{~cm}$ distal to the ligament of Treitz, at least $10 \mathrm{~cm}$ in length, were obtained in $10 \%$ buffered formalin solution. Written informed consent from the authorized relatives was obtained. After receiving in the laboratory, the samples were grossed for length, color of serosa, presence of perforation, hemorrhage, fibrosis, scar, exudates, abscesses, sutures, and so forth. Further tissue processing was done automatically on Histokinette (Leica Biosystems, Germany) automated tissue processing unit and final embedding in paraffin wax was done on Tissue-Tek (Sakura Finetek, United States) semiautomated apparatus. Thin sections of 3 to $4 \mu \mathrm{m}$ were obtained by sectioning the embedded sample on semiautomated microtome. The sections were put in a tissue flotation bath and then taken on slides.

- Staining for light microscopy for jejunum histology: For light microscopy, sections were taken on Superfrost slides (Fisher Scientific, United States). Then the slides were warmed to 60 to $70^{\circ} \mathrm{C}$ for 2 minutes on the slide warming table. This fixed the tissue to the slides. They were stained with hematoxylin and eosin stain. The light microscopic findings were evaluated by three observers based on presence or absence of thrombosis, ulceration, and inflammation, and on the intensity of thinning and/or infarction of wall. Each sample was given a score out of 7 as per - Table 1 (also refer to - Fig. 1).

- Staining for immunohistochemistry for AQ4 and NPY in jejunum: For immunohistochemistry the sections were taken on aminopropyltriethoxysilane (APES) solution-coated Polysine microslides. These sections were run on BenchMark ULTRA automated immuno-histo chemistry/in-situ hybridization (IHC/ISH) slide staining system (Ventana Medical Systems, Inc., F. Hoffmann-La Roche Ltd; automated immunohistochemistry machine) for staining for AQ4 and NPY. The following are the steps that were performed automatically in the system. Antigen retrieval was done using cell conditioning 1 buffer in three steps. First conditioning was done for 8 minutes at room temperature, followed by mild conditioning for 30 minutes, and finally standard conditioning for 60 minutes. For AQ4, 1:100 dilutions of AQ4 primary antibodies were applied and incubated overnight at

Table 1 Scoring of microscopic findings

\begin{tabular}{|l|l|l|l|}
\hline \multirow{2}{*}{ Finding } & \multicolumn{3}{|c|}{ Score } \\
\cline { 2 - 4 } & 0 & 1 & 2 \\
\hline $\begin{array}{l}\text { Presence of } \\
\text { thrombosis }\end{array}$ & Absent & Present & - \\
\hline $\begin{array}{l}\text { Presence of } \\
\text { ulceration }\end{array}$ & Absent & Present & - \\
\hline $\begin{array}{l}\text { Presence of } \\
\text { inflammation }\end{array}$ & Absent & Present & - \\
\hline Thinning of wall & Nil & Mild/moderate & Severe \\
\hline Infarction of wall & Nil & Mild/moderate & Severe \\
\hline
\end{tabular}



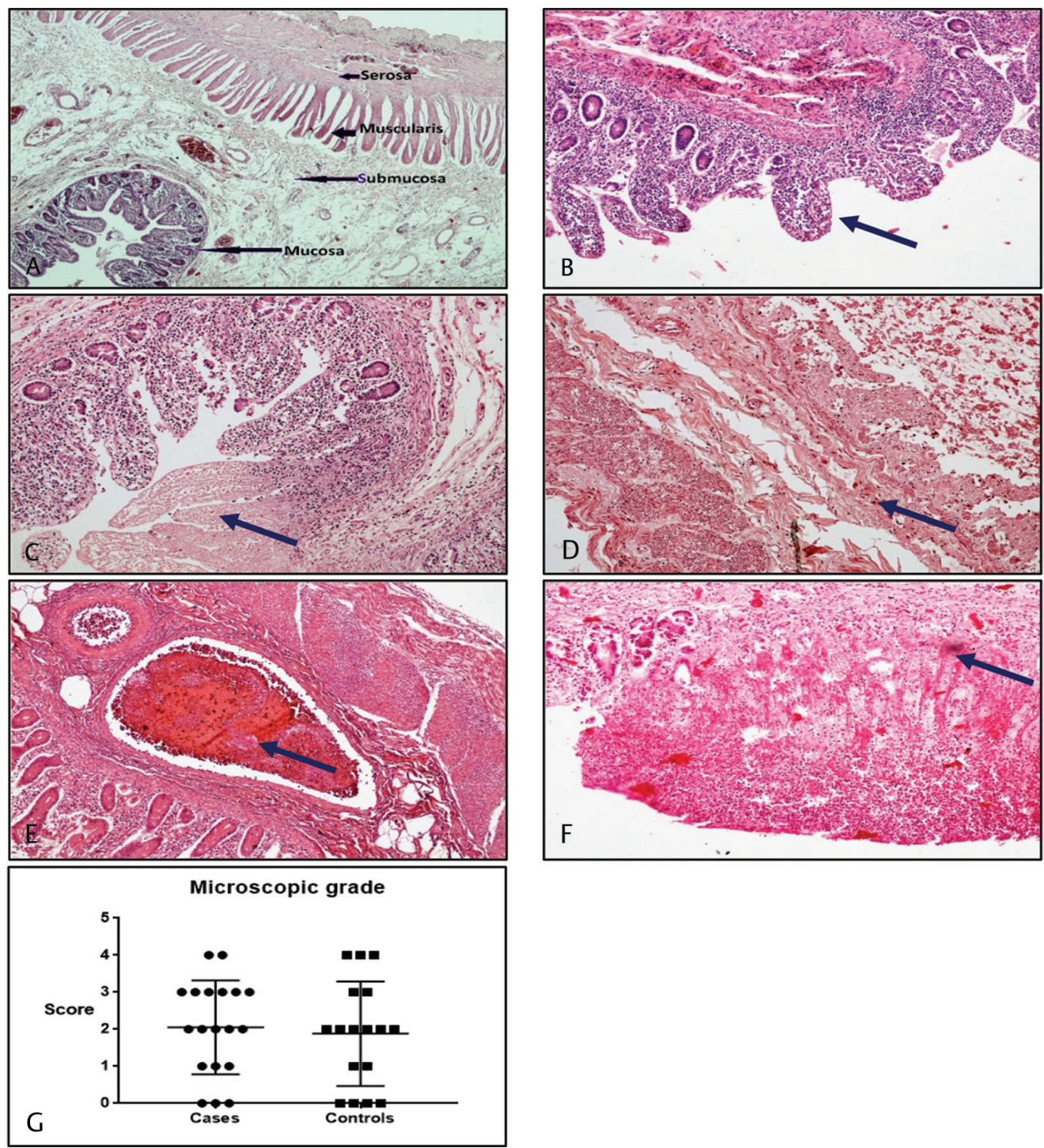

Fig. 1 Histopathological (light microscopic) changes in the jejunal mucosa. (A) Normal morphology of the human jejunal mucosa; H\&E stain at $10 \times$ power. (B) Presence of inflammation (arrow) seen in the jejunal mucosa; H\&E stain at 10×. (C) Infarction (arrow) seen in the jejunal mucosa; H\&E stain at 10x power. (D) Thinning of the wall (arrow) seen in the jejunal mucosa; H\&E stain at $10 \times$ power. (E) Presence of thrombosis (arrow) seen in the jejunal mucosa; H\&E stain at $10 \times$ power. (F) Presence of ulceration (arrow) seen in the jejunal mucosa; H\&E stain at $10 \times$ power. (G) Scatter plot showing individual values of cases and controls along with the mean and standard deviation.

$4^{\circ} \mathrm{C}$ followed by washing thrice with $1 \mathrm{X}$ PBS (phosphate buffered saline). For NPY, 1:600 dilutions of NPY primary antibodies were applied and incubated at $37^{\circ} \mathrm{C}$ for 2 hours followed by washing thrice with $1 \mathrm{X}$ PBS. Respective horseradish peroxidase-conjugated secondary antibody for AQ4 and NPY at 1:400 dilutions was applied and washing was done thrice followed by addition of chromogenic substrate (diaminobenzedine $[D A B])$. Slides were dried and then analyzed microscopically.

IHC staining for AQ4 and NPY was evaluated blind by three observers and the score was average for each sample. Slides were examined for the presence or absence of staining, location of cells showing a positive reaction, and the intensity of positivity of the reaction in the cells. Accordingly, the slides were graded as $0,1,2,3$, and 4 , with 0 indicating no reaction and $1,2,3$, and 4 indicating increasing grades of positivity.

\section{Results}

A total of 40 autopsy samples were obtained for the study which included 20 cases and 20 controls. One case had to be excluded from the analysis as focused assessment with sonography for trauma (FAST) was positive. Similarly, three controls also had to be excluded as one of them was FAST positive, one had a retroperitoneal hematoma, and one had thoracic and abdominal injury but FAST could not be traced. So, a total of 19 cases and 17 controls were analyzed in the study. Among the controls, nine were patients who were brought dead (53\% controls approximately). The results are summarized in the flowchart in $\boldsymbol{- F i g} \mathbf{2}$. 


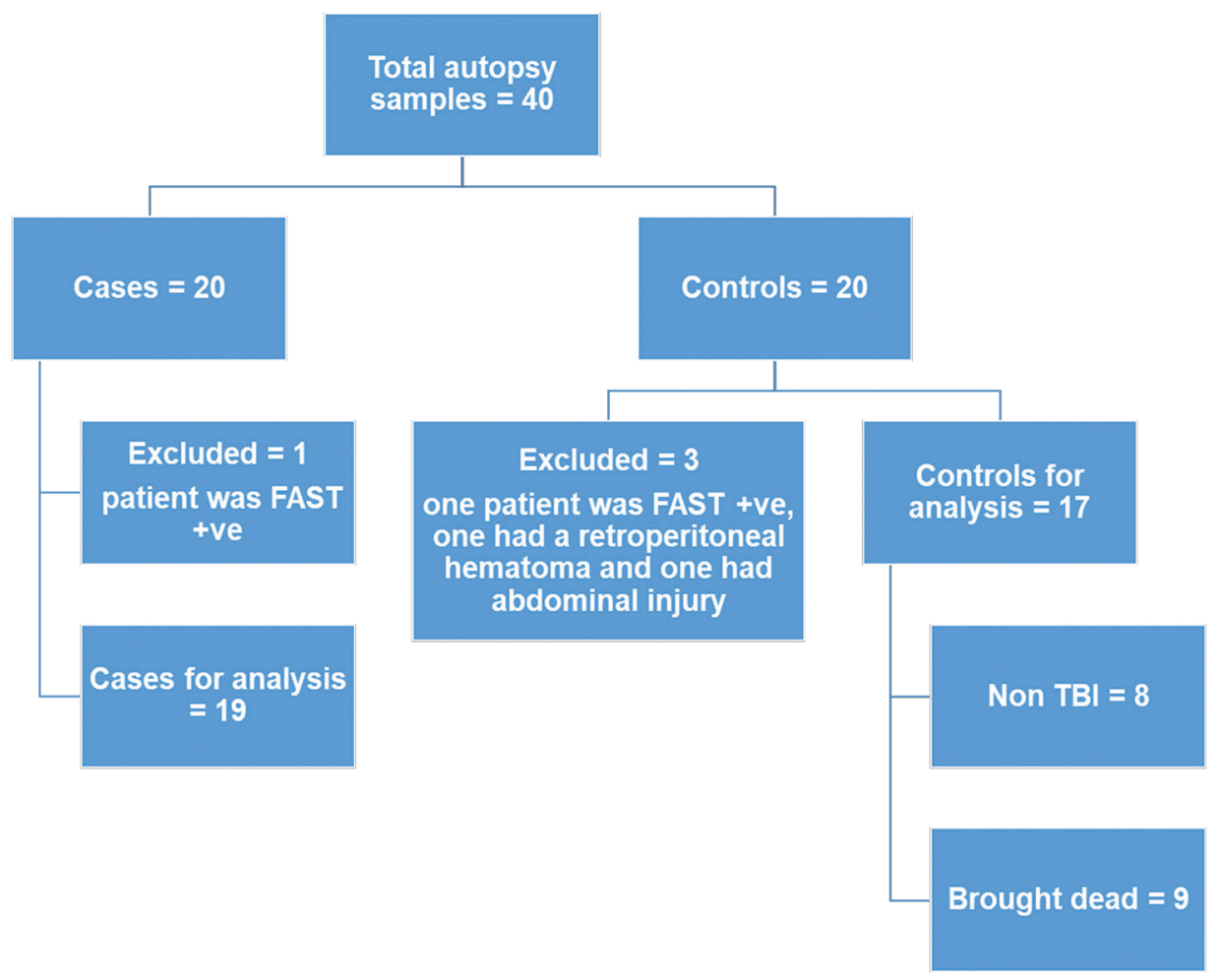

Fig. 2 Flowchart of overall case and control selection. FAST, focused abdominal sonography in trauma; TBI, traumatic brain injury.

\section{Demographics}

The median age of the cases was 35 years (range: 19-75 years) and that of the controls was 39 years (range: 18-75 years). Males outnumbered females in both cases and controls (16 males against 3 females in cases; 15 males against 2 females in controls). The leading cause of trauma was road traffic accidents (13/19 in cases and $9 / 17$ in controls). The other causes were fall from height, railway track accidents, gunshot injury to head, and so forth. The time from injury to the death of the patient was calculated in days. The time from injury to death ranged from 1 to 73 days in cases and from 0 to 86 days in controls. The glasgow coma score (GCS) and injury severity score (ISS) were evaluated at the time of admission to grade the severity of injury. The mean GCS was decreased in both the cases and the controls, which was statistically insignificant (cases, $6.36 \pm 0.75$; controls, $7.52 \pm 1.32$; $p$-value $=0.43$ ). The mean ISS was significantly higher in the controls than the cases (cases, $31.16 \pm 2.32$; controls, $52.18 \pm 5.65 ; p$-value 0.001). The GCS was decreased and the ISS were raised in the cases. Almost half of the controls were patients who were brought dead, in whom the GCS was lowest and the ISS was highest. Only two cases had evidence of sepsis. None of the controls had sepsis.

\section{Gross Findings}

The samples were grossed for length, color of serosa, perforation, hemorrhage, fibrosis, scar, exudates, abscesses, sutures, and so forth. Samples were labeled as
Table 2 Distribution of gross findings in cases and controls

\begin{tabular}{|l|l|l|l|}
\hline \multirow{2}{*}{} & \multicolumn{3}{|c|}{ Gross findings } \\
\cline { 2 - 4 } & Yes & No & Total \\
\hline Case & 9 & 10 & 19 \\
\hline Controls & 8 & 9 & 17 \\
\hline Total & 17 & 19 & 36 \\
\hline
\end{tabular}

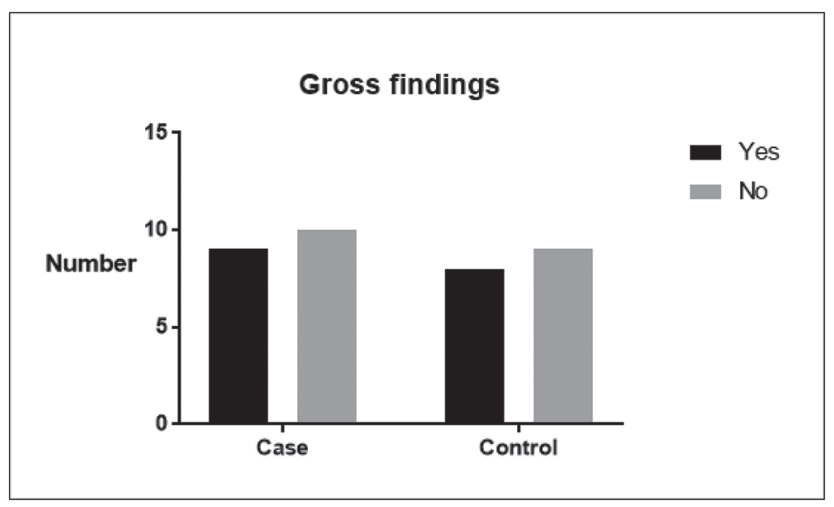

Fig. 3 Bar diagram showing the occurrence of gross examination findings among the cases and the controls.

"YES," indicating significant gross examination finding and "NO," indicating a normal sample or a sample with no significant gross findings; 9 cases out of 19 and 8 controls out of 17 had gross findings. The results obtained are depicted in - Table 2 and - Fig. 3 . The odds ratio was 1.01 , which 
suggests that head injury has a role to play, but a chi-square test used for analysis indicated that the gross examination findings were not significantly different between the cases and the controls ( $p$-value $>0.99$ ). This indicates that gross findings can be present post trauma in individuals, but it cannot reliably differentiate between post TBI and other causes. The individual details of cases and controls have been enumerated in the - Table 3 .

Table 3 Distribution of gross, microscopic and immunohistochemical expression of AQ4 and NPY in individual cases and controls

\begin{tabular}{|c|c|c|c|c|c|c|c|c|c|}
\hline & \multirow{2}{*}{$\begin{array}{l}\text { Gross } \\
\text { findings }\end{array}$} & \multicolumn{6}{|c|}{ Microscopic findings score } & \multirow{2}{*}{$\begin{array}{l}\mathrm{AQ4} \\
\text { intensity } \\
\text { score }(0-4)\end{array}$} & \multirow{2}{*}{$\begin{array}{l}\text { NPY } \\
\text { intensity } \\
\text { score (0-4 }\end{array}$} \\
\hline & & $\begin{array}{l}\text { Thrombosis } \\
(0-1)\end{array}$ & $\begin{array}{l}\text { Ulceration } \\
(0-1)\end{array}$ & $\begin{array}{l}\text { Inflammation } \\
(0-1)\end{array}$ & $\begin{array}{l}\text { Thinning } \\
(0-2)\end{array}$ & $\begin{array}{l}\text { Infarction } \\
(0-2)\end{array}$ & $\begin{array}{l}\text { Total } \\
(0-7)\end{array}$ & & \\
\hline \multicolumn{10}{|l|}{ Cases } \\
\hline 1 & NO & 0 & 1 & 1 & 1 & 0 & 3 & 2 & 2 \\
\hline 2 & YES & 1 & 1 & 1 & 1 & 0 & 4 & 2 & 4 \\
\hline 3 & YES & 0 & 0 & 1 & 0 & 1 & 2 & 2 & 3 \\
\hline 4 & YES & 0 & 0 & 0 & 0 & 0 & 0 & 2 & 3 \\
\hline 5 & YES & 0 & 0 & 0 & 1 & 2 & 3 & 2.5 & 1 \\
\hline 6 & NO & 1 & 0 & 1 & 0 & 0 & 2 & 2 & 4 \\
\hline 7 & YES & 0 & 1 & 1 & 0 & 0 & 2 & 2 & 3 \\
\hline 8 & NO & 0 & 1 & 1 & 1 & 0 & 3 & 2.5 & 4 \\
\hline 9 & YES & 1 & 1 & 0 & 2 & 0 & 4 & 2 & 2.5 \\
\hline 10 & NO & 1 & 1 & 0 & 1 & 0 & 3 & 2 & 3 \\
\hline 11 & NO & 0 & 0 & 0 & 0 & 0 & 0 & 3 & 2.5 \\
\hline 12 & NO & 0 & 0 & 0 & 0 & 0 & 0 & 2 & 1.5 \\
\hline 13 & NO & 0 & 1 & 1 & 0 & 0 & 2 & 2 & 1.5 \\
\hline 14 & YES & 0 & 1 & 1 & 1 & 0 & 3 & 3 & 2.5 \\
\hline 15 & NO & 0 & 0 & 1 & 0 & 0 & 1 & 2.5 & 1.5 \\
\hline 16 & YES & 1 & 1 & 1 & 0 & 0 & 3 & 2.5 & 1 \\
\hline 17 & NO & 0 & 0 & 1 & 0 & 0 & 1 & 2 & 1 \\
\hline 18 & NO & 1 & 0 & 0 & 0 & 0 & 1 & 3 & 1 \\
\hline 19 & YES & 1 & 0 & 1 & 0 & 0 & 2 & 3.5 & 1 \\
\hline Mean & Not applicable & & & $2.05 \pm 0.29$ & & & & $2.34 \pm 0.10$ & $2.26 \pm 0.24$ \\
\hline \multicolumn{10}{|c|}{ Controls } \\
\hline 1 & YES & 1 & 1 & 1 & 1 & 0 & 4 & 3 & 3.5 \\
\hline 2 & YES & 1 & 0 & 1 & 1 & 0 & 3 & 0.5 & 2 \\
\hline 3 & YES & 0 & 0 & 1 & 0 & 1 & 2 & 3 & 3 \\
\hline 4 & NO & 0 & 1 & 1 & 0 & 0 & 2 & 2 & 2.5 \\
\hline 5 & NO & 1 & 0 & 0 & 0 & 0 & 1 & 0.5 & 1.5 \\
\hline 6 & NO & 1 & 0 & 1 & 0 & 0 & 2 & 3 & 2 \\
\hline 7 & NO & 0 & 0 & 0 & 0 & 0 & 0 & 2 & 1 \\
\hline 8 & NO & 0 & 0 & 0 & 0 & 0 & 0 & 2 & 1 \\
\hline 9 & YES & 1 & 0 & 1 & 2 & 0 & 4 & 1 & 3 \\
\hline 10 & NO & 0 & 1 & 1 & 0 & 0 & 2 & 1 & 3 \\
\hline 11 & NO & 0 & 0 & 0 & 0 & 0 & 0 & 1 & 3 \\
\hline 12 & NO & 1 & 1 & 1 & 0 & 0 & 3 & 1 & 2 \\
\hline 13 & YES & 0 & 1 & 0 & 0 & 0 & 1 & 2.5 & 3 \\
\hline 14 & YES & 1 & 0 & 1 & 1 & 1 & 4 & 3 & 2.5 \\
\hline 15 & YES & 0 & 0 & 0 & 0 & 0 & 0 & 1 & 1.5 \\
\hline 16 & YES & 0 & 1 & 1 & 0 & 0 & 2 & 1.5 & 1 \\
\hline 17 & NO & 0 & 1 & 0 & 1 & 0 & 2 & 3 & 2.5 \\
\hline Mean & Not applicable & & & $1.88 \pm 0.34$ & & & & $1.82 \pm 0.23$ & $2.23 \pm 0.19$ \\
\hline$p$-value & $>0.99$ & & & 0.7 & & & & 0.04 & 0.93 \\
\hline
\end{tabular}




\section{Microscopic Findings}

Changes in the gut mucosa from normal were searched for in the study. For doing this the standard H\&E staining was done. We have graded these microscopic changes on a score of 0 to 7 based on the presence or absence of thrombosis in the mucosa, or ulceration and inflammation in the mucosa. Infarction of the mucosal wall and the thinning of the wall secondarily caused due to it were also noted; 16 out of $19(\sim 84 \%)$ cases and 13 out of $17(\sim 76 \%)$ controls had changes in the jejunal mucosa, and 7 out of 19 cases and 7 out of 17 controls had the presence of thrombosis. Similarly, 9 cases and 7 controls had ulceration and 12 cases and 10 controls had the presence of inflammation; 7 cases and 5 controls had evidence of mucosal wall thinning, among which 1 case and 1 control had severe wall thinning. Mucosal wall infarction was seen in two cases and two controls, and one case had severe wall infarction. The mean microscopy score of cases was $2.05 \pm 0.29$ and that of controls was $1.8 \pm 0.34$. An unpaired $t$-test was used to for comparison. Although microscopic findings were present in both the cases and the controls, they were not significant statistically ( $p$-value $=0.70$ ). The findings of individual cases and controls are illustrated in -Table $\mathbf{3}$ and - Fig. 1.

\section{Immunohistochemical Findings}

AQ4 and NPY expression in the jejunal mucosa was evaluated by immunohistochemistry on a score of 0 to 4 , with 0 indicating no expression. The mean intensity of AQ4 positivity in the cases group was $2.34 \pm 0.10$ and in the control group was $1.82 \pm 0.23$. An unpaired $t$-test was used to compute the difference between the two groups. This difference in the intensity was statistically
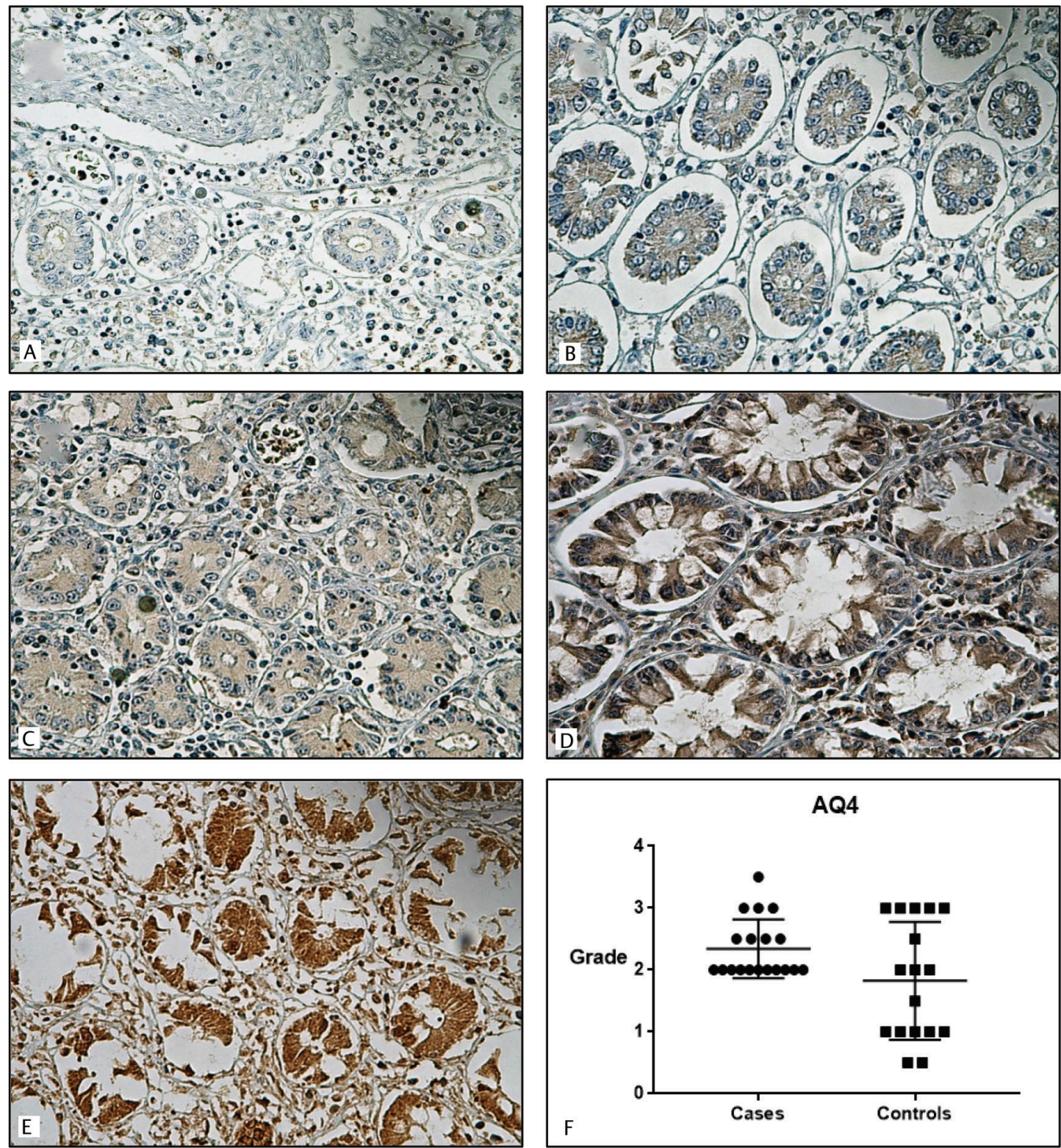

Fig. 4 The categorization of aquaporin 4 by immunohistochemistry of paraffin-embedded human jejunal tissue with anti-aquaporin 4 antibody (Abcam ab11026) at 1:100 dilution. (A) Grade 0: Nil positivity, at 40× power. (B) Grade 1: Mild positivity, at 40× power. (C) Grade 2: Moderate positivity, at 40× power. (D) Grade 3: High positivity, at 40× power. (E) Grade 4: Intense positivity, at 40× power. (F) Scatter plot showing individual values of cases and controls along with the mean and standard deviation. 

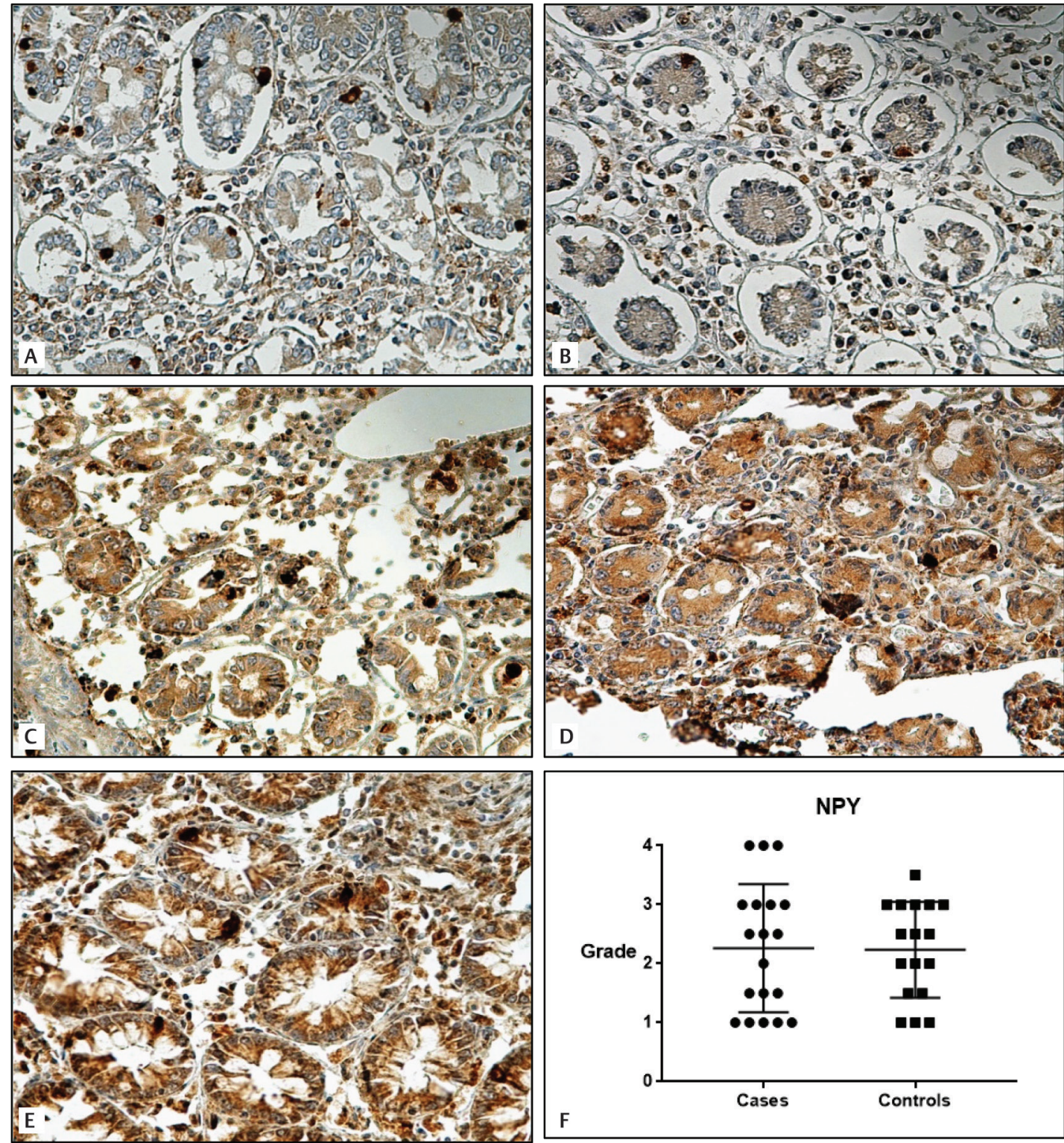

Fig. 5 The categorization of neuropeptide $Y$ by immunohistochemistry of paraffin-embedded human jejunal tissue with anti-neuropeptide $Y$ antibody (Abcam ab180809) at 1:600 dilution. (A) Grade 0: Nil positivity, at 40× power. (B) Grade 1: Mild positivity, at 40× power. (C) Grade 2: Moderate positivity, at 40× power. (D) Grade 3: High positivity, at 40× power. (E) Grade 4: Intense positivity, at 40× power. (F) Scatter plot showing individual values of cases and controls along with the mean and standard deviation.

significant with a $p$-value of 0.04 , indicating that AQ4 was expressed more in the cases than the controls. The expression of AQ4 in cases and controls are enumerated in - Table 3 and -Fig. 4. The mean intensity of NPY expression in the cases group was $2.26 \pm 0.24$ and in the control group was $2.23 \pm 0.19$. An unpaired $t$-test used to compute the difference between the two groups did not reveal any statistically significant difference ( $p$-value $=0.93$ ). The expression of NPY in cases and controls are enumerated in - Table 3 and - Fig. 5 .

\section{Discussion}

India being a rapidly developing country is prone to vehicular congestion on roads which is a major factor for RTA, which in turn is a common cause of TBI. As most vehicles in India are driven by males, they are more likely to be involved in RTA than females. This same scenario was encountered in our study as RTA was the most common cause of TBI and the incidence of RTA was more in males than females by a significant fraction.

GI dysfunction is known to complicate the prognosis in patients of TBI, mainly due to a dysfunction in the brain-gut axis which is formed by the hypothalamus-pituitary-adrenal axis and the autonomic nervous system. It is responsible for regulating the intestinal functions, in health as well as in physiologically compromised states.6.11 Dysfunction in this axis results in a myriad of complications like vomiting, gastric reflux, delayed gastric emptying, intolerance to enteral feeding to reduced intestinal peristalsis, enterogenous sepsis, and so forth. ${ }^{12}$ Various brain-gut peptides like cholecystokinin (CCK), vasoactive intestinal peptide (VIP), 
calcitonin gene-related peptide (CGRP), neuropeptide $\mathrm{Y}$ (NPY), and substance P (SP) are known to influence the intestinal mucosa and affect the function and integrity of remote organs and tissues, leading to systemic inflammatory response syndrome and multiple organ dysfunction syndromes (MODS). ${ }^{13}$

Studies in animal models under controlled conditions $7,14,15$ have been done to demonstrate the GI changes post TBI, but the same cannot be done in humans as it would be inhumane to subject humans to such agonizing and life-threatening trauma. So we designed a study to study the same in patients dying due to TBI. However, a person could have several pre-existing conditions before the TBI which could also cause GI changes. To overcome this difficulty a thorough research of the medical history was undertaken by referring to the medical records, drug history, and by talking to the relatives and attendants of the deceased. Also patients who underwent any GI intervention were not included in the study. Utmost care was taken not to include those patients with significant medical history or conditions that could induce a bias in the study. As per our understanding, this study design could thus prove useful to understand the GI changes post TBI in humans.

GCS is a system developed to access the conscious state of a human with the help of three parameters: eye opening, verbal response, and motor response; the maximum score being 15 , which indicates a fully conscious person and a minimum score of 3 indicates unconscious and unresponsive person. It has been traditionally used by health care workers to access and monitor patients with head trauma and patients in intensive care unit (ICU) settings. The mean score at the time of admission was decreased in both the groups. A decrease in GCS is expected in the cases group as they are the ones with TBI. Also, it was the lowest in the patients who were brought dead.

ISS is a scoring system to access the severity of trauma. The score was raised in the control group because of highest score among the patients who were brought dead. This score was statistically significant but because of the skewed results no significant inference could be drawn.

The specimens received for histopathology were inspected under direct vision for morphology and other significant findings. No significant difference between cases and controls was found ( $p$-value $>0.99$ ), which indicates some alteration in the morphology of adults can happen irrespective of TBI. Changes like inflammation, thrombosis, ulceration along with thinning, and infarction of the intestinal wall that cannot be always discovered by gross examination need light microscopy. ${ }^{7}$ Hence, a score based on this was formulated to grade the severity of the changes. This score was high in both the cases and controls ( $p$-value $=0.70$ ). Thus, both the groups can be assumed to be comparable as the gross and microscopic findings were not different between cases and controls. It would be wise to infer that confounding bias of pre-existing or undetected mucosal pathology was removed to some extent by these findings. This also means that histopathological examination could not be used as a standalone test for the assessment of GI changes in TBI patients, thus implying the necessity of a marker, something which could show a significant difference in expression among the cases and the controls.

Studies in animals have shown AQ4 and NPY to be raised in jejunal mucosa post TBI. ${ }^{9}$ NPY, a 36 amino acid-long polypeptide, is one of the most potent orixegenic peptide in the body.$^{16}$ In the digestive tract, NPY inhibits GI motility and electrolyte secretion, and in this way modify the input to the brain. ${ }^{8}$ On the other hand aquaporins, which are charged integral membrane proteins, regulate water molecule entry across the cell and block the passage of other ions and solute. ${ }^{17}$ They are related to many diseases like diabetes insipidus, salivary gland dysfunction (Sjogren's syndrome), cataracts, and even hypertension. A major function of GI tract of fluid transport is believed to be regulated by AQ4, and so it is accepted that it plays a role in several GI tract related diseases as well. ${ }^{18}$ Thus, AQ4 and NPY have been postulated to play a role in the pathogenesis of intestinal dysfunction after TBI. Increased NPY levels may be responsible for intestinal ischemia and hypoxia, whereas AQP4 may play an important role in intestinal edema. The expression of both AQ4 and NPY has been studied in animals under controlled conditions and found to be raised and significantly different in TBI. ${ }^{9} \mathrm{We}$ studied the expression of these two markers, AQ4 and NPY, by immunohistochemistry. Our study revealed a significantly raised expression of AQ4 in jejunum post TBI ( $p$-value $=0.04$; - Table 3). However, the same was not true for the expression of NPY ( $p$-value $=0.93 ;-$ Table 3 ). The exact reason for this difference between the findings between animal studies and this study could not be ascertained.

\section{Conclusion}

TBI has a deep impact on the GI morphology and functioning, and ultimately on the prognosis. Traditional methods like histopathological examination cannot be used to distinguish between the changes occurring in the jejunal mucosa post TBI and post non-TBI. Expression of AQ4 in the jejunum post TBI is increased, as demonstrated by immunohistochemistry, and it can be used as a marker of GI injury of TBI in humans. The value of NPY expression, although increased post TBI in controlled studies in animals, needs further evaluation in humans. This study is novel by being the one of the few to explore the secondary changes in human jejunum induced by TBI. Further studies will be required to correlate the intensity of expression with the intensity of TBI.

Limitations of the study: As stated time and again, isolated TBI in humans, wherein the most common cause of TBI being road traffic accidents, is an idealistic condition in real world. And unfortunately, it cannot be artificially induced in the 
laboratory. So, the possibility of an unknown and undiagnosed injury always lurked around in the background.

The controls we used were diseased controls as they had some form of trauma or the other except TBI and abdominal injury. So, comparison with healthy age-matched controls could not be done.

\section{Note}

This study was presented at 3rd AIIMS Neurotrauma Conference (ANTC), October 28-31, 2015.

\section{Funding}

This study was funded by All India Institute of Medical Sciences.

\section{Conflict of Interest}

None declared.

\section{Acknowledgment}

We would like to acknowledge Ms. Venencia Albert, PhD scholar, for helping us with the standardization and running of IHC tests, data compilation, and statistics.

\section{References}

1 Yattoo G, Tabish A. The profile of head injuries and traumatic brain injury deaths in Kashmir. J Trauma Manag Outcomes 2008;2(1):5

2 Pilitsis JG, Rengachary SS. Complications of head injury. Neurol Res 2001;23(2-3):227-236

3 Feighery L, Smyth A, Keely S, et al. Increased intestinal permeability in rats subjected to traumatic frontal lobe percussion brain injury. J Trauma 2008;64(1):131-137, discussion 137-138

4 Katzenberger RJ, Ganetzky B, Wassarman DA. The gut reaction to traumatic brain injury. Fly (Austin) 2015;9(2):68-74

5 Ma EL, Smith AD, Desai N, et al. Bidirectional brain-gut interactions and chronic pathological changes after traumatic brain injury in mice. Brain Behav Immun 2017;66:56-69
6 Bansal V, Costantini T, Kroll L, et al. Traumatic brain injury and intestinal dysfunction: uncovering the neuro-enteric axis. J Neurotrauma 2009;26(8):1353-1359

7 Hang CH, Shi JX, Li JS, Wu W, Yin HX. Alterations of intestinal mucosa structure and barrier function following traumatic brain injury in rats. World J Gastroenterol 2003;9(12): 2776-2781

8 Holzer P, Reichmann F, Farzi A. Neuropeptide Y, peptide YY and pancreatic polypeptide in the gut-brain axis. Neuropeptides 2012;46(6):261-274

9 Duan H, Hao C, Fan Y, et al. The role of neuropeptide Y and aquaporin 4 in the pathogenesis of intestinal dysfunction caused by traumatic brain injury. J Surg Res 2013;184(2):1006-1012

10 Zhu C, Chen Z, Jiang Z. Expression, distribution and role of aquaporin water channels in human and animal stomach and intestines. Int J Mol Sci 2016;17(9):E1399

11 Mayer EA. Gut feelings: the emerging biology of gut-brain communication. Nat Rev Neurosci 2011;12(8):453-466

12 Norton JA, Ott LG, McClain C, et al. Intolerance to enteral feeding in the brain-injured patient. J Neurosurg 1988;68(1):62-66

13 Godlewski J, Pidsudko Z, Sienkiewicz W. Concentration of vasoactive intestinal polypeptide, neuropeptide $\mathrm{Y}$ and calcitonin gene related peptide in neoplastic tissue of the human large intestine. Contemp Oncol 2011;15(2):65-68

14 Hang CH, Shi JX, Li JS, Li WQ Wu W. Expressions of intestinal NF-kappaB, TNF-alpha, and IL-6 following traumatic brain injury in rats. J Surg Res 2005;123(2):188-193

15 Chen G, Shi JX, Qi M, Wang HX, Hang CH. Effects of progesterone on intestinal inflammatory response, mucosa structure alterations, and apoptosis following traumatic brain injury in male rats. J Surg Res 2008;147(1):92-98

16 Tatemoto K. Neuropeptide Y: complete amino acid sequence of the brain peptide. Proc Natl Acad Sci USA 1982;79(18): 5485-5489

17 Sabolić I, Valenti G, Verbavatz JM, et al. Localization of the CHIP28 water channel in rat kidney. Am J Physiol 1992; 263(6 Pt 1):C1225-C1233

18 Koyama Y, Yamamoto T, Tani T, et al. Expression and localization of aquaporins in rat gastrointestinal tract. Am J Physiol 1999;276(3):C621-C627 